\title{
Induction thermography as an alternative to conventional NDT methods for forged parts
}

\author{
by P. BOUTEILLE*, G. LEGROS*
}

\author{
*CETIM, NDT Division, 52 avenue Félix Louat, 60300 Senlis, France, patrick.bouteille@cetim.fr
}

\begin{abstract}
Dye penetrant inspection and magnetic particle inspection are widely used in industry, to inspect metallic components. These non-destructive testing methods are highly efficient but they induce possible risks for operators' health (use of chemicals, exposure to solvent vapors and to electromagnetic fields, etc.) and for the environment (generation of effluents, significant energy consumption for drying or magnetization operations, etc.).

As they are faced with tougher environmental rules, increasing numbers of manufacturers take a real interest in environmentally-friendly alternative inspection methods capable of giving similar results. CETIM, the French technical center for the mechanical industry, has been involved in the development of active infrared thermography for several years, especially induction thermography on forged components.

After tests on a car ball joint previously published, we will present a comparison between induction thermography and magnetic particle inspection on a batch of 26 cracked wheel hubs. Destructive metallographic analyses confirm that induction thermography is able to reveal more defects which are invisible to the naked eye than magnetic particle inspection, yet considered as the reference method. A second application of induction thermography will be presented on non-magnetic components. Tests results on artificial hip joints made of titanium, austenitic steel and cobalt-based alloy will be presented in comparison with dye penetrant inspection results.

Finally, the advantages and drawbacks of induction thermography will be discussed compared to those of magnetic particle inspection and dye penetrant inspection.
\end{abstract}

\section{Introduction}

Forging consists in forming a malleable material by impact or pressing. On the one hand, this method has the advantage of being performed at a temperature less than the melting temperature of the material. On the other hand, it makes it possible to form a part while locally improving its mechanical properties. This is why this method is widely used in the automobile industry to manufacture ball joints and wheel hubs and in the medical industry to manufacture prostheses. During the forging process, various defects, such as cold shuts or cracks can appear on the produced part. For obvious safety reasons, for example in the automobile and medical industries, it is absolutely necessary to inspect $100 \%$ of the parts produced. Then the inspection methods generally used are magnetic particle inspection for magnetic materials and dye penetrant inspection for non-magnetic materials:

Magnetic particle inspection consists in generating an intense magnetic flux inside a ferromagnetic material and then in observing the field lines on its surface using a magnetic particle product which contains colored or fluorescent particles. If a defect is present, these field lines are disturbed. The analysis of these signatures allows the surface or subsurface defects to be detected. Dye penetrant inspection consists in applying a penetrant liquid which contains colored or fluorescent markers onto the surface of the part to be inspected. The liquid penetrates by capillarity into all open defects. After removal of the excess penetrant, a developer is then applied onto the examined part. The developer sucks the liquid which remains in the cracks like a blotting paper and reveals them. Therefore, dye penetrant inspection allows detection of open defects only.

Magnetic particle inspection and dye penetrant inspection are very efficient and easy-to-use non-destructive testing methods. They have thus become widely used in the industrial sector. Unfortunately, they have a few drawbacks. On the one hand, they require the use of cleaning solvents and aqueous-based or petroleum-based products (red or fluorescent penetrant products plus developers for dye penetrant inspection, magnetic products for magnetic particle inspection). These products are not very environmentally-friendly. On the other hand, the drying, magnetization and demagnetization operations carried out on the parts require large amounts of energy. Cleaning of parts and removal of the excess penetrant generate a lot of effluents which will have to be treated. And at last, these two methods have a significant impact on operators' health. As a matter of fact, operators are exposed to solvent vapors, organic products and electromagnetic fields. It would be interesting to reduce these drawbacks or even introduce, in the forging industry, new non-destructive testing methods which would not have such drawbacks.

The new European Directives aim at reducing the use of energy-intensive, waste-generating methods potentially hazardous to operators' health. Increasing numbers of industrialists have become aware of the constraints associated with conventional testing methods and they try to turn to alternative, more environmentally-friendly methods capable of giving comparable results. This explains why CETIM has been studying for several years the possibilities of infrared thermography coupled with induction excitation for non-destructive testing of forged parts.

The results obtained with induction thermography on steel wheel hubs and on titanium, stainless steel and cobalt alloy artificial hip joints are presented herein. 


\section{Inspection of a ferromagnetic steel wheel hub}

\subsection{Presentation of wheel hubs}

The wheel hub is the central section of a wheel attached to the shaft on bushing side, and on which the rim is installed, on wheel centering side. The wheel hub is a safety component made of ferromagnetic steel with an outside diameter of $130 \mathrm{~mm}$ and a height of approximately $70 \mathrm{~mm}$.

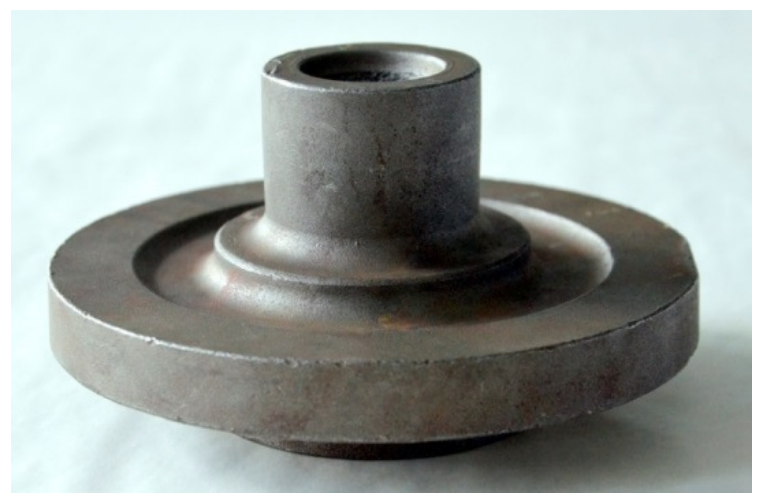

Figure 1: Tested wheel hub

In the automobile industry, the studied component is submitted to a full magnetic particle inspection on an automated line. The decision is then made by an operator on line output.

\section{$2.2 \quad$ Testing conditions}

Concerning infrared thermography, two inspection configurations have been developed in order to cover the entire wheel hub. The upper section (bushing side) and the lower section (wheel centering side) of the wheel hub are successively inspected, using the same circular coil. The hub circumference is inspected through 4 lateral acquisitions (with a $90^{\circ}$ rotation of the hub) with two concentric coils. Therefore, 6 acquisitions are necessary to inspect a full wheel hub. The acquisition is carried out with a $100 \mathrm{~Hz}$ cooled camera, FLIR SC7600. Acquisition time is 400 ms, including heating and cooling.

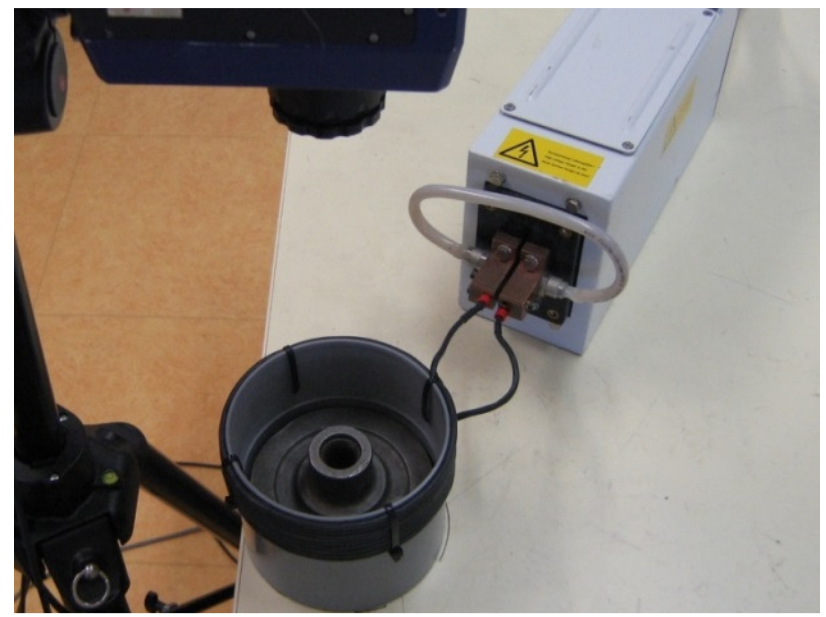

Figure 2: Thermography inspection on the wheel hub

\subsection{Comparison between thermography inspection and magnetic particle inspection}

In order to assess the detection performance of induction thermography versus a reference method, 26 wheel hubs with defects were subjected to magnetic particle inspection and thermography inspection. Metallographic sections were made on some indications in order to check for a defect and characterize it.

The pictures below represent phase images corresponding to thermography inspections of those wheel hubs. As a comparison, we also display the results of the magnetic particle inspection carried out on these same hubs.

An indication on the bushing of the hub below is detected by magnetic particle inspection (Figure 3 , right-hand side) as well as by thermography with a side view (Figure 3, left-hand side). 

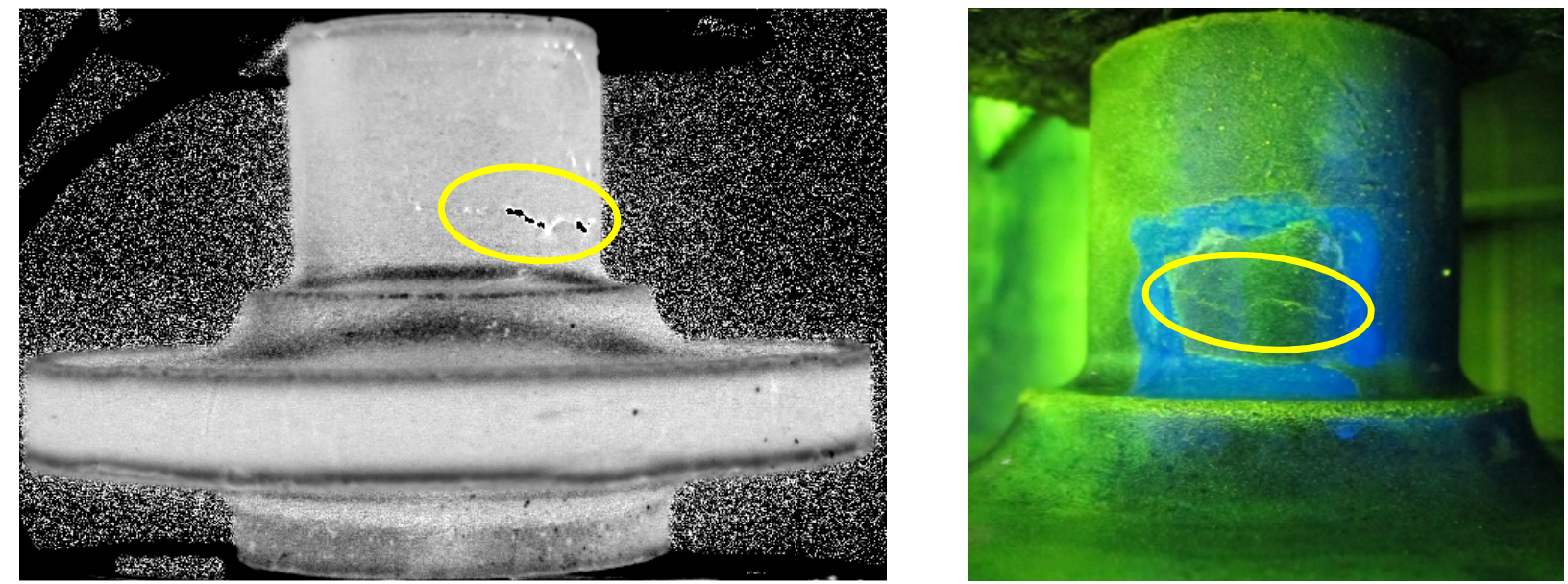

Figure 3: Circumferential indication detected by thermography (left) and by magnetic particle inspection (right)

A metallographic section made on this indication confirms the presence of a rather deep cold shut.

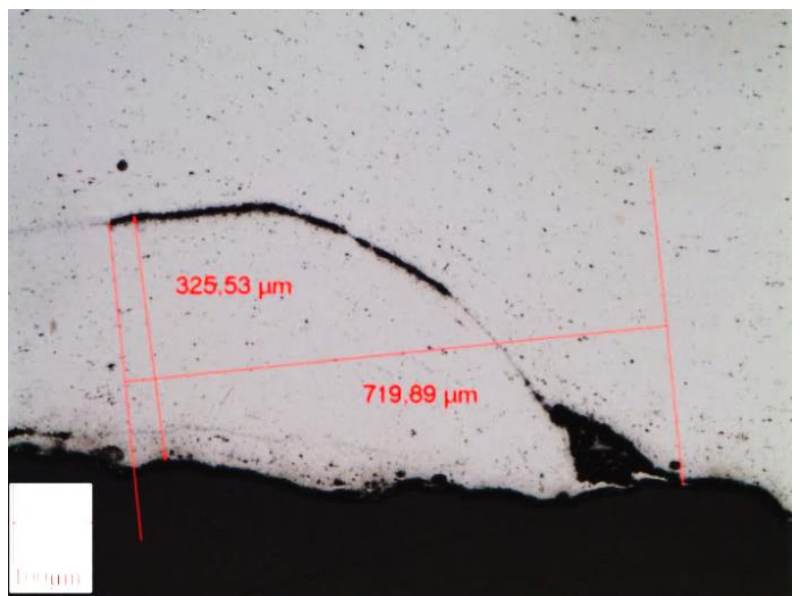

Figure 4: Metallographic analysis on the indication detected by thermography and magnetic particle inspection

Figures 5 show a radial defect detected both by infrared thermography (left) and magnetic particle inspection (right) on wheel centering side.
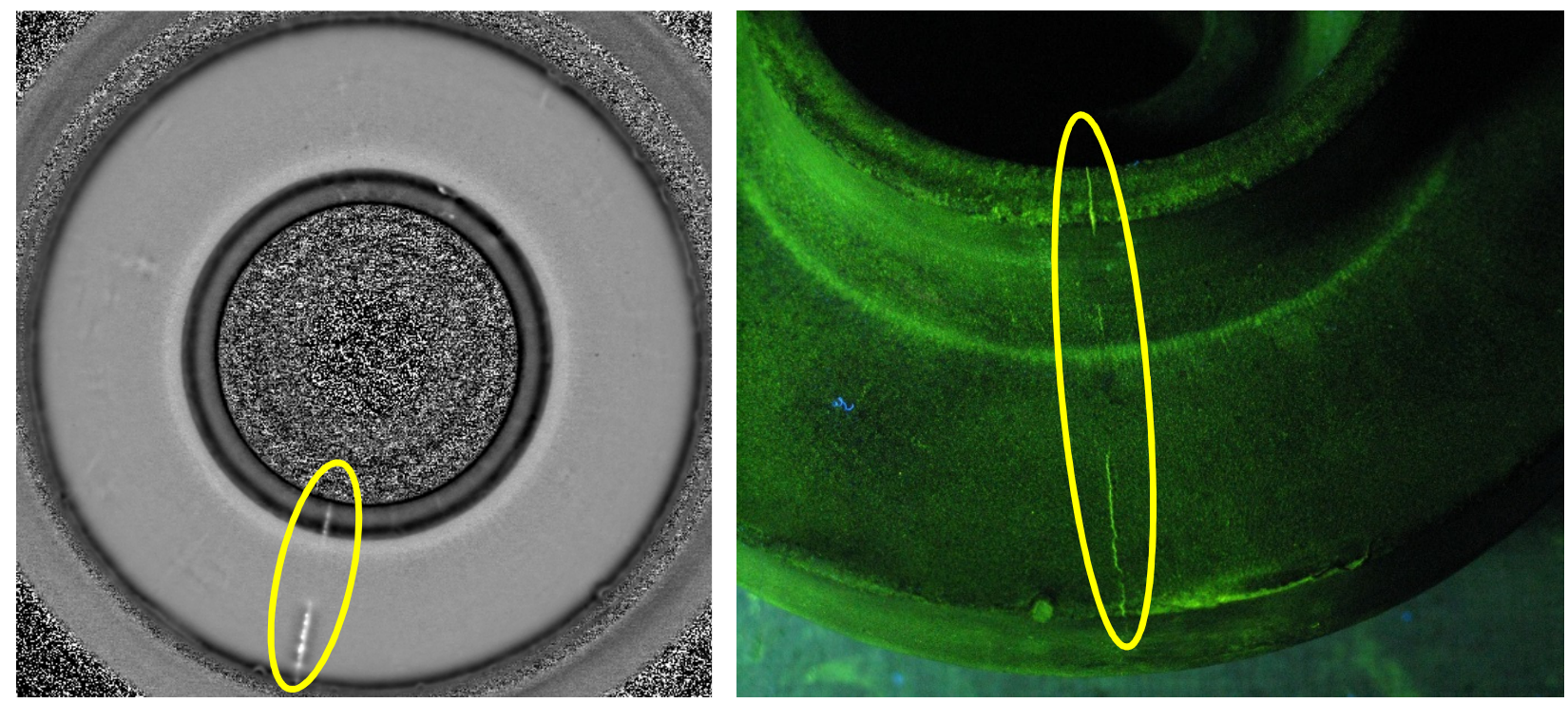

Figure 5: Radial indication detected by thermography (left) and by magnetic particle inspection (right) 
The same coil and the same infrared thermography configuration simultaneously evidenced the radial and circumferential indications. This is confirmed on the following phase image obtained during the thermography inspection of a hub with radial and circumferential defects. The circumferential defect (number 2) is hardly detected by magnetic particle inspection, in spite of the choice of a testing configuration conducive to its detection.
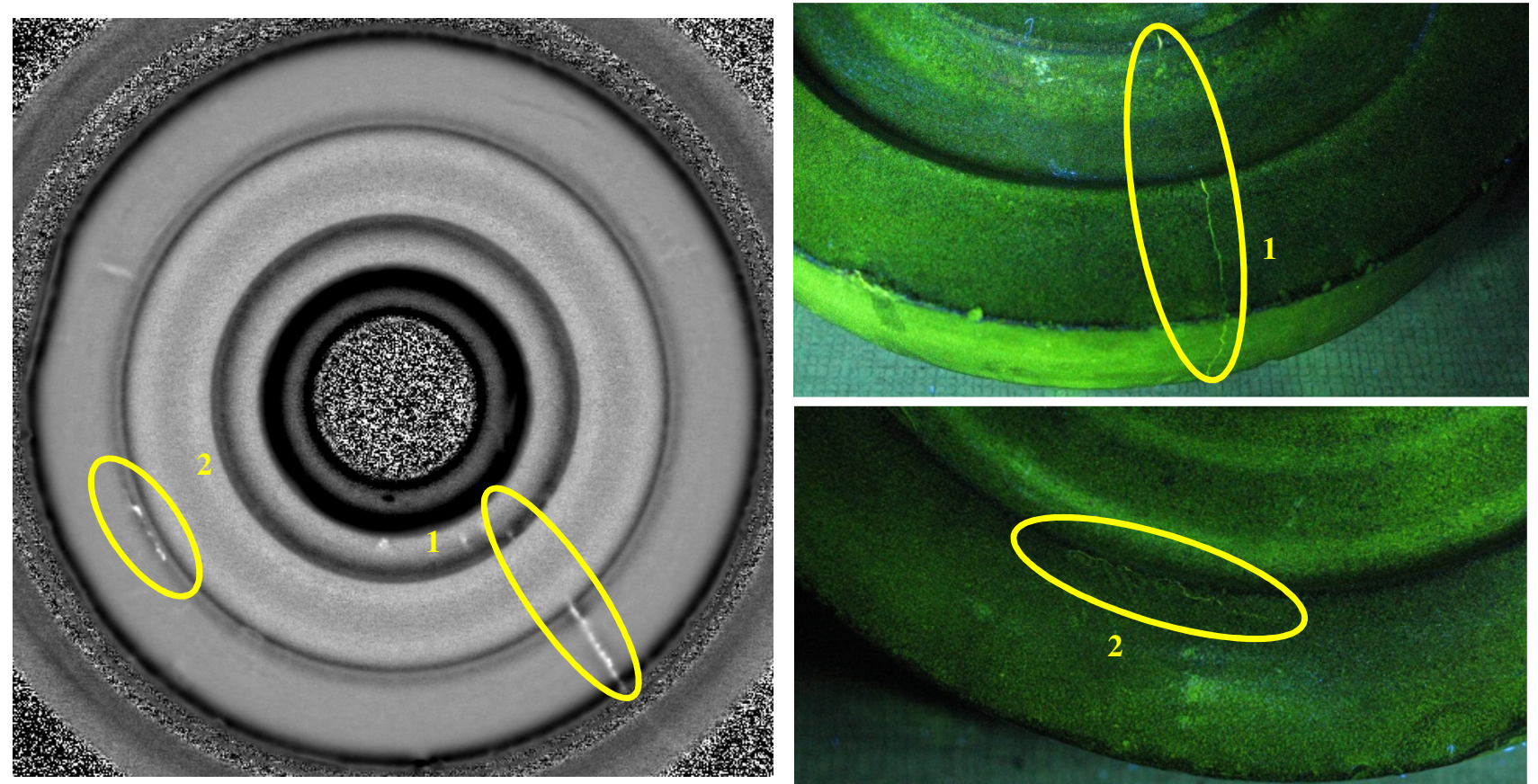

Figure 6: Inspection on bushing side by thermography (left) and magnetic particle inspection (right)

On Figure 7, induction thermography revealed three circumferential indications on bushing side. The circumferential indication numbered 3 is barely detected by magnetic particle inspection although it is well detected by induction thermography.
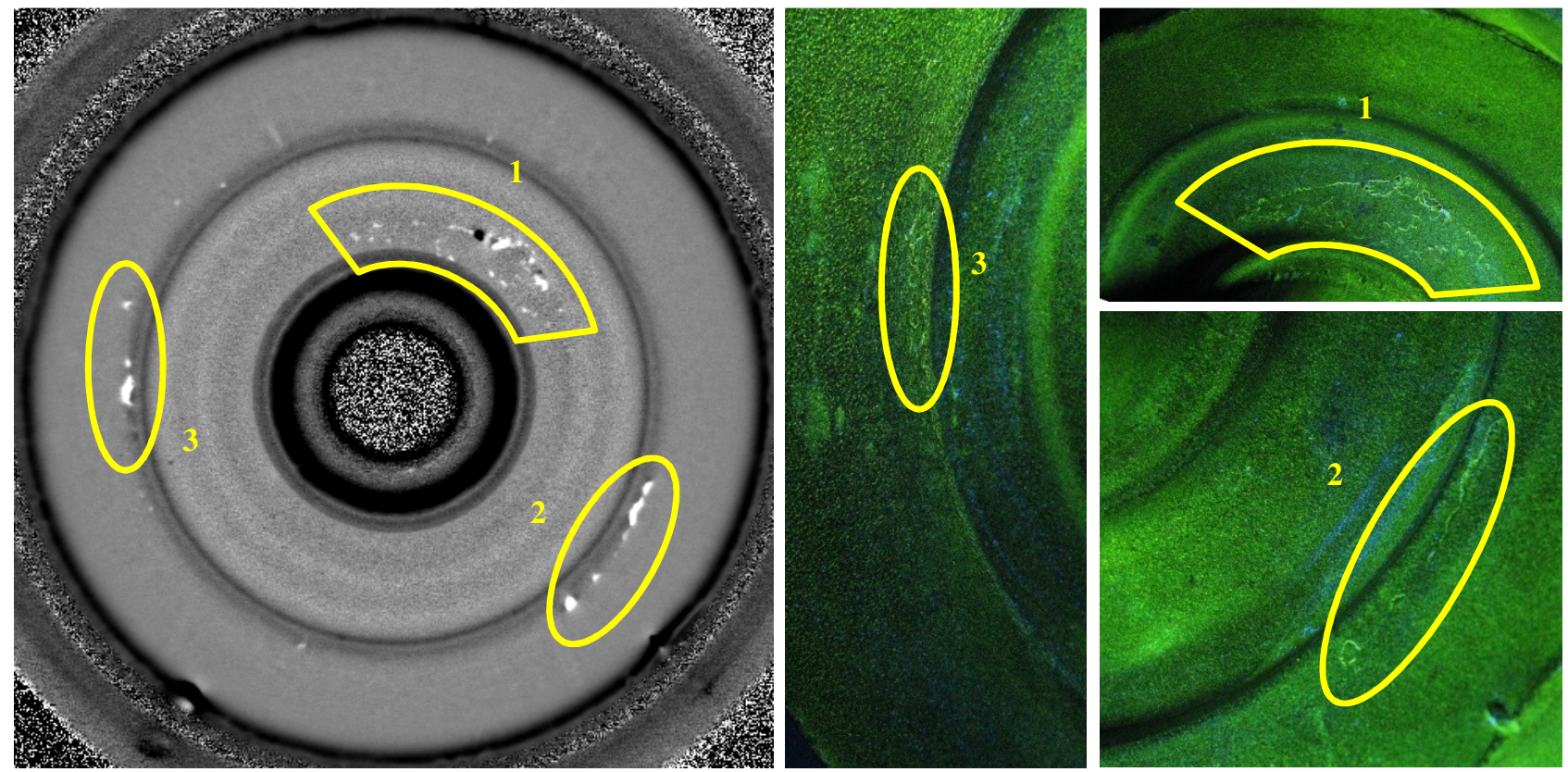

Figure 7: Inspection on bushing side by thermography (left) and by magnetic particle inspection (right)

A metallographic section was made on this indication which was very hardly detected by magnetic particle inspection. This section confirmed the presence of a cold shut at the indication detected by thermography (Figure 8). 


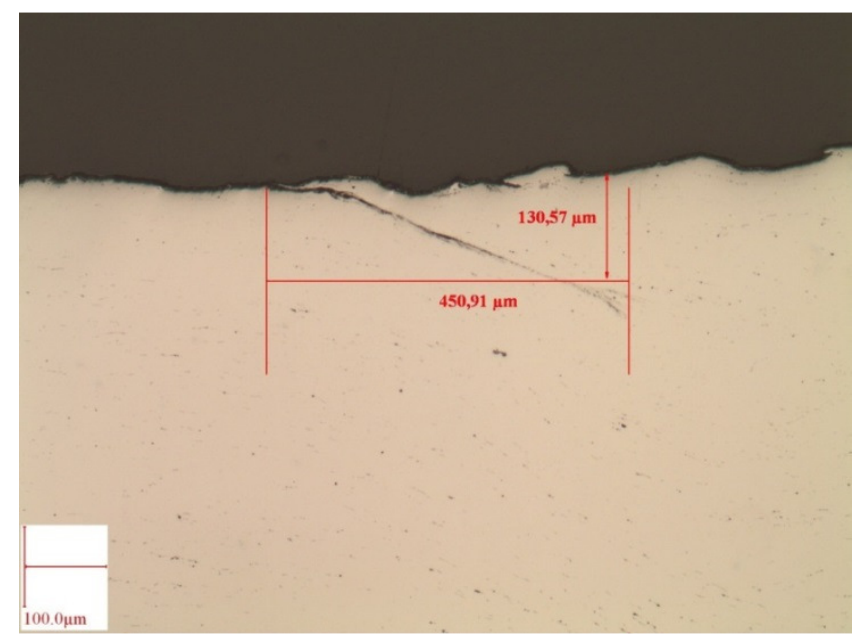

Figure 8: Metallographic analysis on an indication detected by thermography but not detected by magnetic particle inspection

\subsection{Conclusion on this case}

The concentration of eddy currents around the defects reveals very thin defects although the resolution of the camera is not sufficient to see them in the visible spectrum. Therefore, on the hubs, the indications seem to have a width of approximately one millimeter whereas the opening of the defect is only of a few microns.

Thermography allows defects to be detected in both directions (radial and circumferential) even if the chosen configuration is better suited for radial defects.

Over the 26 wheel hubs with defects subjected to infrared thermography and magnetic particle inspection, thermography revealed more indications than magnetic particle inspection which is yet considered as a reference method in the industrial sector. The metallographic analyses carried out on approximately twenty indications confirmed the presence of a defect for each detected indication.

In order to cover the entire hub, 6 acquisitions are necessary, with rotation or the turning over of the part. An automated inspection method would allow each hub to be inspected within a few seconds. It can provide an inspection rate similar to that of the current industrial magnetic particle inspection process.

Obtaining digital images also allows the sanction to be automated: it allows computer-aided detection of indications on images obtained by thermography. This step would make it possible to reduce the manpower necessary for the inspection and, thereby, to reduce the costs of the inspection.

Therefore, infrared thermography coupled with induction excitation is a credible alternative for the replacement of magnetic particle inspection on this type of forged parts.

\section{Inspection of an artificial hip joint made of non-magnetic material}

\subsection{Presentation of the hip joint}

Artificial hip joints with defects were entrusted to CETIM by a forging company specializing in orthopedic equipment.

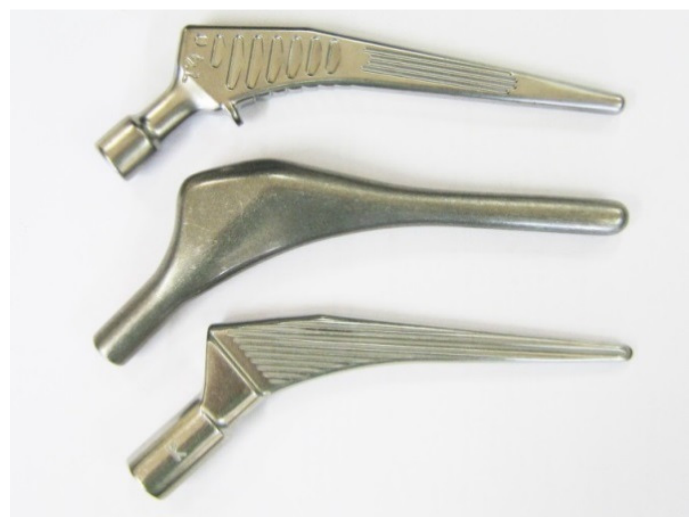

Figure 9: Tested artificial hip joints 
They are $100 \%$ dye penetrant inspected during the industrial process. These smooth or grooved prostheses are made of various non-magnetic materials:

- $\quad$ Titanium alloy, TA6V-ELI

- Cobalt-chromium alloy, CoCrMo

- $\quad$ Stainless steel, M30NW

\section{2 $\quad$ Testing conditions}

As the tested prostheses were made of non-ferromagnetic materials, induction heating was less efficient for infrared thermography inspection on these parts compared to ferromagnetic parts. As a matter of fact, the depth of the eddy currents is more significant on these materials.

The tested inductors are composed of two Helmholtz coils, i.e. two circular coils with the same radius, parallel to each other and located opposite each other and at a distance equal to the coil radius. Electrical current is circulated in these coils and the magnetic field created in their vicinity is relatively uniform at the center of the system.

Acquisition is carried out with a $100 \mathrm{~Hz}$ cooled camera, FLIR SC7600.

\subsection{Comparison between thermography testing and penetrant testing}

Infrared thermography is assessed through tests carried out on 15 artificial hip joints and comparison with the results obtained with penetrant testing.

Figures 10 show a phase image corresponding to the thermography testing (left) of a smooth stainless steel prosthesis and the result of the penetrant testing (right) carried out on this same prosthesis.
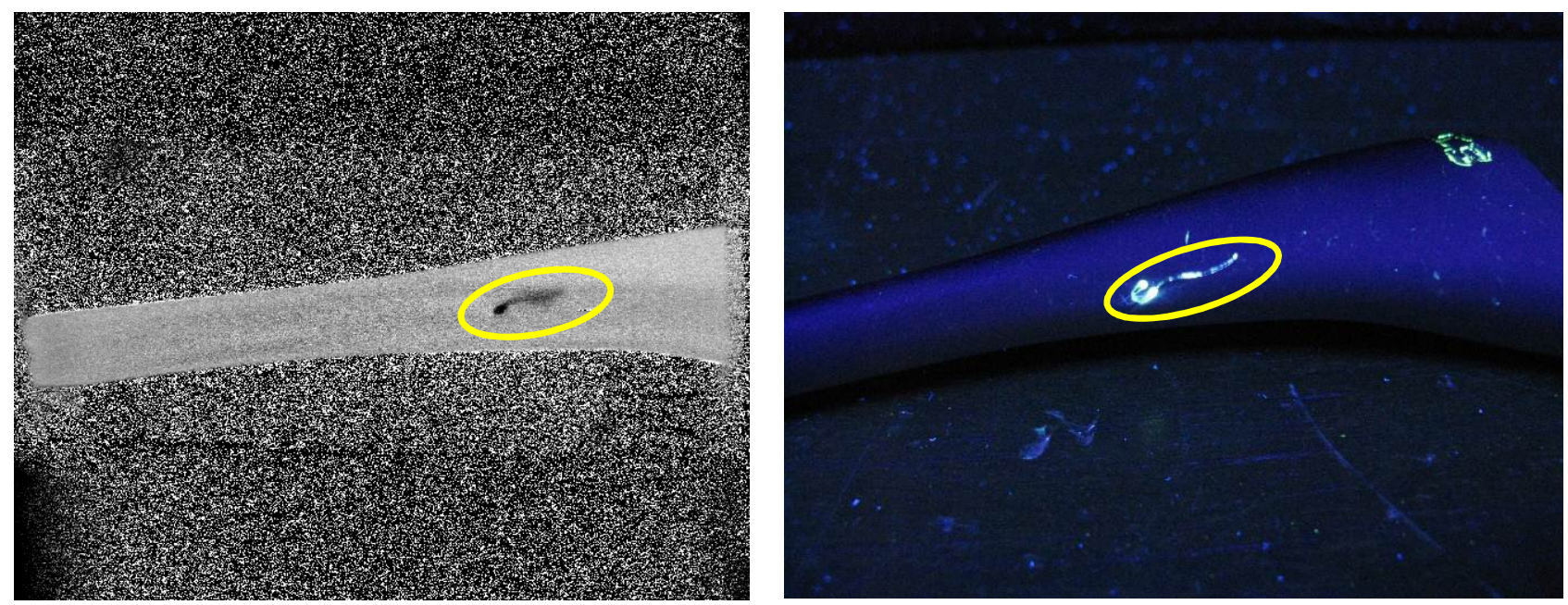

Figure 10: Inspection of a stainless steel artificial hip joint by thermography testing (left) and penetrant testing (right)

The figures below show the comparison between thermography testing and penetrant testing in order to detect a defect on the smooth portion of a cobalt-chromium alloy prosthesis.
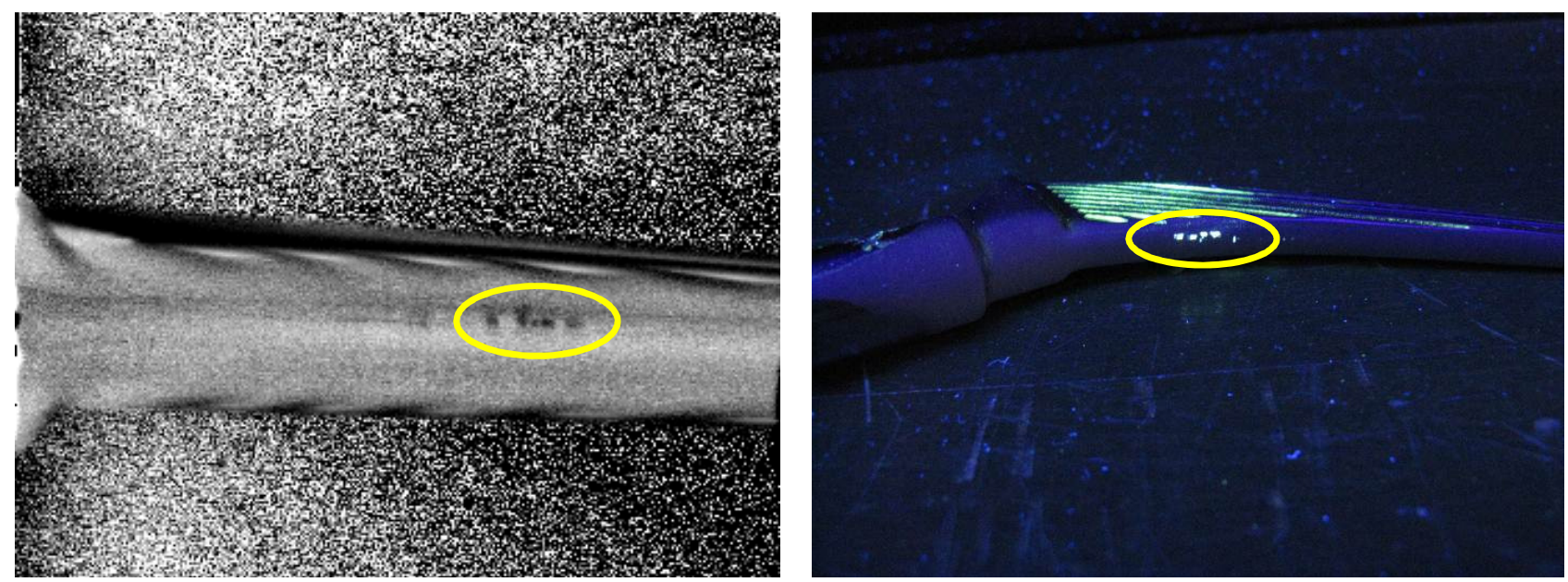

Figure 11: Inspection of a cobalt-chromium alloy prosthesis by thermography testing (left) and penetrant testing (right) 
Forging defects located inside a groove on titanium prostheses aren't detected by this thermography configuration, although they are detected by penetrant testing (Figure 12).

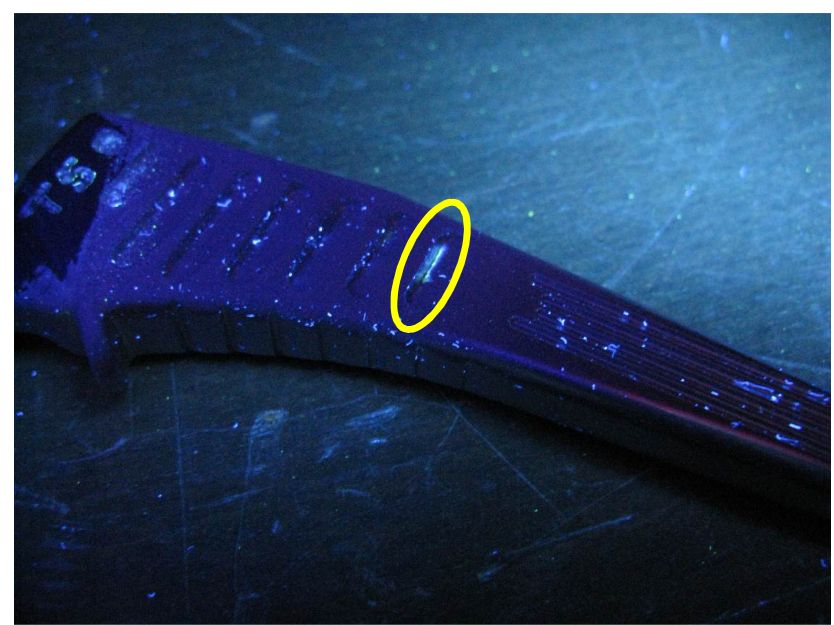

Figure 12: Inspection of a titanium prosthesis by penetrant testing

The position of the defect (at the bottom of a groove) or its depth, are certainly the reasons why it was not detected. In fact, detecting defects on titanium parts is possible. In particular, this is the case in the example below (Figure 13) on a titanium plate with defects created artificially by forging.

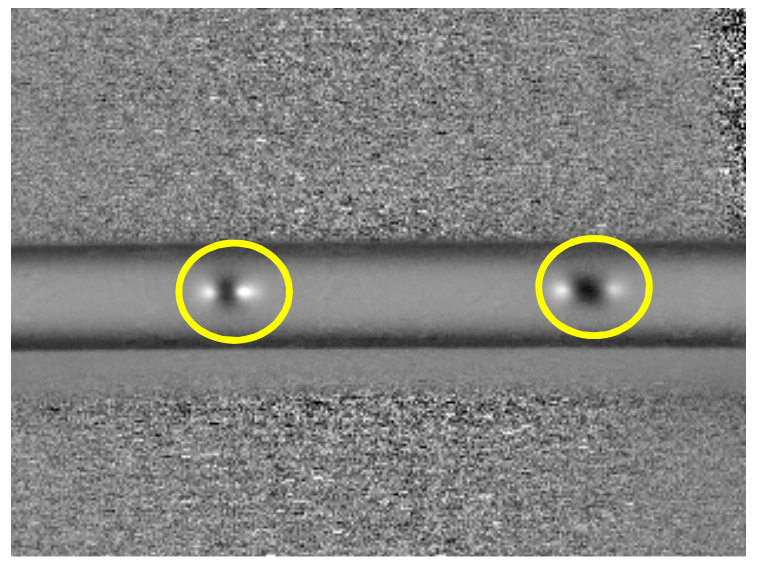

Figure 13: Inspection of a titanium plate by thermography testing (TT)

\subsection{Conclusion on this case}

Induction excitation is well suited to ferromagnetic materials, but it is less efficient at heating titanium, stainless steel or cobalt-based alloy. At equal frequency, the depth of the eddy currents generated by induction is more significant on these materials than on ferromagnetic materials.

However, these tests prove that infrared thermography coupled with induction excitation reveals part of the defects found during penetrant testing. On the artificial hip joints, only the defects located in the grooves are not detected by infrared thermography. The defects located on a smooth area of the prosthesis, invisible with the naked eye, are obviously detected.

\section{CONCLUSIONS AND PERSPECTIVES}

Through its various studies $[5,6,7,8]$, CETIM is trying to better determine the limits of infrared thermography for detecting surface defects on metallic materials.

In this document, we have proven that induction thermography is a possible alternative for the replacement of magnetic particle inspection on magnetic forged parts such as wheel hubs. In fact, in this case, thermography makes it possible to obtain the same detection quality as conventional testing methods. The testing rate is similar, with an inspection of a few seconds after automation. Automatic sanction, i.e. automatic detection of defects without human intervention is very difficult with magnetic particle testing but it is possible with thermography testing thanks to the digital images provided by cameras. This represents a significant reduction of inspection costs. Thermography is also an environmentally-friendly, energy-efficient method which does not require the use of any chemical. Finally, thermography 
reduces risks for personnel's health as it complies with European directives regarding operators' exposure to electromagnetic fields and solvent vapors.

We have also demonstrated that induction thermography reveals forging defects on non-magnetic materials. Comparisons with penetrant testing on hip prostheses are satisfactory. Eddy current generation at higher frequencies would make it possible to limit the current penetration depth and maybe improve detection. Making metallographic sections at the detected defects in order to assess their depth and opening would also make it possible to draw conclusions on the performance and limits of thermography for non-magnetic materials.

\section{REFERENCES}

[1] Maldague X., "Introduction to NDT by active infrared thermography", Materials Evaluation, Vol. 6, No 9, pp. 1060 1073, 2002

[2] Bodnar J.L., Edée M., Menu C., Besnard R., Le Blanc A., Pigeon M., Sellier J.Y., "Cracks detection by a moving photothermal probe", Journal de Physique IV, C7-592, 1994

[3] Zweschper Th., Dillenz A., Riegert G., Scherling D., Busse G., "Ultrasound excited thermography using frequency modulated elastic waves", Insight, Vol. 45, No 3, 2003

[4] Vrana J., Goldammer M., Baumann J., Rothenfusser M., Arnold W., "Mechanisms and Models for Crack Detection with Induction Thermography", Review of Progress in QNDE 27, pp. 475-482, 2008

[5] Maillard S., Cadith J., Eschimese D., Walaszek H, Mooshofer H., Candore J. C., Bodnar J.L., "Towards the use of passive and active infrared thermography to inspect metallic components in the mechanical industry", QIRT congress proceedings, Laval (Quebec), 2010

[6] Maillard S., Cadith J., Walaszek H., Bodnar J.L., "Active infrared thermography and its new applications to metallic materials", COFREND congress proceedings, Dunkerque (France), 2011

[7] Bouteille P., Legros G., Maillard S., Cadith J., Bodnar J.L., "Induction active thermography as an alternative to magnetic particle inspection", QIRT congress proceeding, Naples (Italy), 2012

[8] Bouteille P., Legros G., "Induction thermography: an alternative to conventional NDT on forged components", COFREND congress proceedings, Bordeaux (France), 2014 